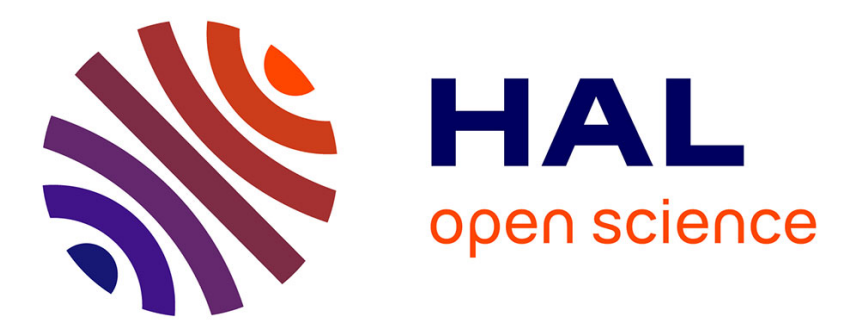

\title{
Coherent polarization rotation of TeraHertz spintronic emitters with in-plane uni-axial magnetic anisotropy
}

Geoffrey Lezier, Pierre Koleják, Jean-Francois Lampin, Kamil Postava, Mathias Vanwolleghem, Nicolas Tiercelin

\section{- To cite this version:}

Geoffrey Lezier, Pierre Koleják, Jean-Francois Lampin, Kamil Postava, Mathias Vanwolleghem, et al.. Coherent polarization rotation of TeraHertz spintronic emitters with in-plane uni-axial magnetic anisotropy. CLEO: Applications and Technology, 2021, San Jose, United States. pp.JM3G.2, 10.1364/CLEO_AT.2021.JM3G.2 . hal-03329137

\section{HAL Id: hal-03329137 \\ https://hal.science/hal-03329137}

Submitted on 30 Aug 2021

HAL is a multi-disciplinary open access archive for the deposit and dissemination of scientific research documents, whether they are published or not. The documents may come from teaching and research institutions in France or abroad, or from public or private research centers.
L'archive ouverte pluridisciplinaire HAL, est destinée au dépôt et à la diffusion de documents scientifiques de niveau recherche, publiés ou non, émanant des établissements d'enseignement et de recherche français ou étrangers, des laboratoires publics ou privés. 


\title{
Coherent polarization rotation of TeraHertz spintronic emitters with in-plane uni-axial magnetic anisotropy
}

\author{
Geoffrey Lezier ${ }^{1}$, Pierre Koleják ${ }^{1,2}$, Jean-François Lampin ${ }^{1}$, Kamil Postava ${ }^{2}$, \\ Mathias Vanwolleghem ${ }^{1}$ and Nicolas Tiercelin ${ }^{1}$ \\ ${ }^{1}$ Univ. Lille, CNRS, Centrale Lille, Univ. Polytechnique Hauts-de-France, UMR 8520-IEMN,F-59000 Lille, \\ France \\ ${ }^{2}$ VSB-Technical University of Ostrava, Nanotechnology centre and IT4Innovations, 17. listopadu 15, 70833 \\ Ostrava-Poruba, Czech Republic \\ geoffrey.lezier@iemn.fr
}

\begin{abstract}
Spintronic TeraHertz emitters using uni-axial anisotropic $\mathrm{FeCo} / \mathrm{TbCo}_{2} / \mathrm{FeCo}$ ferromagnetic layers allow the polarization control of the $\mathrm{THz}$ emitted wave thanks to the Stoner-Wohlfarth coherent rotation of magnetization when submitted to a magnetic field along hard axis. (C) 2020 The Author(s)
\end{abstract}

\section{Introduction}

The TeraHertz (THz) frequency band (extending from 0.1 to $30 \mathrm{THz}$ ) presents a large number of potential applications in a wide variety of fields, from non-invasive control to spectroscopy, imaging or telecommunication. In this framework, THz time-domain spectroscopy (TDS) methods are powerful phase sensitive tools. However, the current $\mathrm{THz}$ generation technologies based on semiconductors are limited in their applications due to the narrow $\mathrm{THz}$ bandwidth or gaps due to phonon absorption. Typical TDS spectra are cut-off maximally at $6 \mathrm{THz}$. THz spintronic emitters, introduced by Kampfrath et al. [1,2], offer a new approach to break this limit. Exciting a 5d/3d nonmagnetic (NM) /ferromagnetic (FM) multilayer by IR fs-pulses generates a spin-polarized current that upon diffusion in the NM metal undergoes strong spin-orbit coupling. The action of the inverse spin-Hall effect transforms the spin-current into a charge current dipole transversely oriented to the spin direction. The ultrafast relaxation times and absence of phonon absorption bands typical of metals allow the generation of THz pulses Fourier-limited by the IR pulse. Gapless spectra up to $30 \mathrm{THz}$ have been reported. The polarization state is intrinsically linked to the magnetization direction in the FM layer. While optimizations of the THz power have been reported, little work has been done on the control of the THz polarization state, other than by rotating the applied field [3]. Here, we demonstrate for the first time coherent control of the magnetization state of a spintronic emitter and thereby a controlled generation of an arbitrary linear $\mathrm{THz}$ polarization.

\section{Results}

In order to be able to control the polarization direction, our proposed solution is to use a strongly uni-axial magnetically anisotropic ferromagnetic layer in the emitter to replace the classic ferromagnetic layer used in spintronics THz experiments. As is known from magnetics, perfect uniaxial anisotropy allows to coherently rotate the magnetization by varying the applied field ONLY along the hard axis between its saturation values. This is known as Stoner-Wohlfarth rotation. Thus, the considered emitter is a W(2nm)/ $\mathrm{FeCo}(0.5 \mathrm{~nm}) / \mathrm{TbCo}_{2}(0.8 \mathrm{~nm}) / \mathrm{FeCo}(0.5 \mathrm{~nm}) /$ $\mathrm{Pt}(2 \mathrm{~nm})$ stack deposited on a c-cut Sapphire substrate by RF sputtering in a LEYBOLD Z550 equipment. During the growth, a magnetic field is applied in the plane of the substrate. Thanks to the exchange coupling between the layers, the result is a magnetically anisotropic and soft material with low saturation field around 1000e, as it is clearly shown by the Vibrating Sample Magnetometer (VSM) measurements on fig. 1a (dotted lines).

The spintronic emitter was then investigated under a normal incidence transmission configuration of a time domain spectroscopy (TDS) setup, exposed by a $100 \mathrm{fs}$ infrared pulse with a central wavelength of $820 \mathrm{~nm}$. The longitudinal and transverse component of the THz emission were measured by combining two polarizers placed between the emitter and a dipole photoconductive antenna. By setting up the delay line of the TDS setup at the point of maximum $\mathrm{THz}$ emission, it was possible to measure it as a function of the applied field. Both $\mathrm{THz}$ components are shown on fig. 1a (full lines) and are perfectly consistent with the VSM measurements. Moreover, the $3 \mathrm{D}$ plot on fig $1 \mathrm{~b}$ also shows that magnetization clearly rotates in a Stoner-Wohlfarth fashion. The visible jumps are caused by the small angle between hard axis and applied magnetic field.

In a second step, TDS spectra of both THz components were recorded for various values of the magnetic field, 
starting from saturation at 200 to -6 Oersted. As seen of fig. 2, the polarization clearly rotates from vertical to horizontal. As it is possible to control the rotation of magnetization over $360^{\circ}$, so is the polarization angle span.
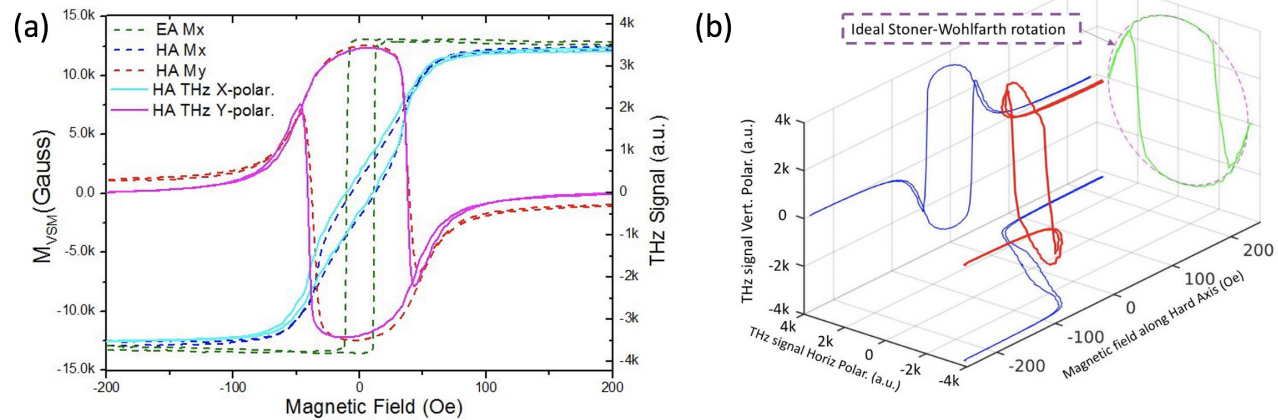

Fig. 1. (a) Comparison between VSM measured magnetic loops (dashed lines) and THz-TDS measurement (full lines) on the maximal peak position. The hysteresis loop with the field applied along the easy axis for the longitudinal component (green) is also presented. (b) 3D representation of the longitudinal and transversal components of the THz emission vs. magnetic field.

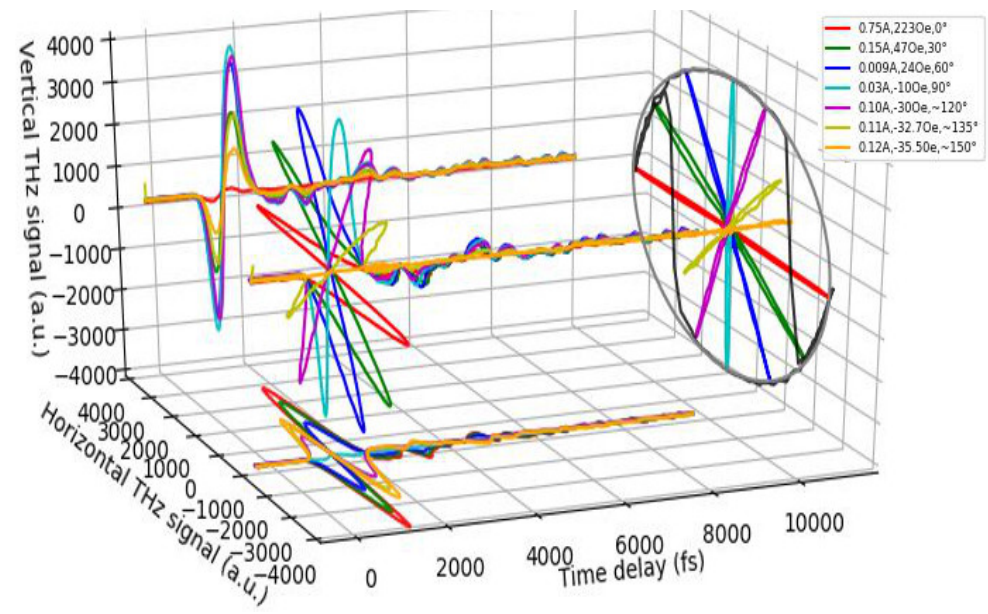

Fig. 2. 3D representation of THz-TDS scan under different magnetic fields to show the rotation of the polarization of the emitted radiation.

\section{Summary}

We present an improved scheme to achieve coherent polarization rotation without multipolar or rotating external magnetic bias nor complex cascaded emitters. This is achieved by exploiting recent progress in artificially engineered strong uniaxial anisotropy in intermetallic heterostructures of rare-earth and transition metals. By replacing the FM layer of the spintronic emitter with a carefully designed $\mathrm{FeCo} / \mathrm{TbCo}_{2} / \mathrm{FeCo}$ heterostructure, we experimentally demonstrated Stoner-Wolfarth-like coherent rotation of the THz polarization only by a unipolar variation of the strength of the hard axis field. These results improve greatly the feasibility of fast polarization switchable integrated $\mathrm{THz}$ sources impacting practical applications such as ultrabroadband $\mathrm{THz}$ spectroscopic ellipsometry without rotating elements, or polarization modulated high speed wireless data communications, but also fundamental physical studies into ultrafast terahertz optospintronics. This works also opens paths toward new $\mathrm{THz}$ high speed modulation schemes via the control of magnetization.

\section{References}

1. T. Seifert, S. Jaiswal, U. Martens, J. Hannegan, L. Braun, P. Maldonado, F. Freimuth, A. Kronenberg, J. Henrizi, I. Radu, et al.,Nat. Photonics., vol. 10, pp. 483, 2016.

2. T. Kampfrath, M. Battiato, P. Maldonado, G. Eilers, J. Nötzold, S. Mährlein, V. Zbarsky, F. Freimuth, Y. Mokrousov, S. Blügel, et al., Nat. Nanotechnol., vol. 8, pp. 256, 2013.

3. Kong, D. and Wu, X. and Wang, B. and Nie, T. and Xiao, M. and Pandey, C. and Gao, Y. and Wen, L. and Zhao, W. and Ruan, C. and Li, Y. and Wang, L., Adv. Opt. Mat., 20(7),pp. 1900487, 2019. 